\title{
Are Tumor Exposure and Anatomical Resection Antithetical during Surgery for Hepatocellular Carcinoma? A Critical Review
}

\section{Guido Torzilli Matteo Donadon Matteo Cimino}

Liver Surgery Unit, Department of General Surgery, University of Milan School of Medicine, Humanitas Cancer Center, Rozzano, Milan, Italy

\section{Key Words}

Cirrhosis · Hepatectomy · Hepatocellular Carcinoma · Intraoperative Ultrasound · Liver

\begin{abstract}
Hepatic resection is the most potentially curative local therapy for patients with hepatocelluar carcinoma (HCC). However, the high rate of postoperative recurrence, 50-70\% at 3 years, remains a major concern. Such recurrences usually occur in the liver owing to the high propensity of HCC to invade the portal vein branches and the underlying liver cirrhosis, which is the ideal background for HCC development. Two pivotal surgical techniques are commonly used to reduce such recurrences: anatomical resection (AR) and achievement of negative margins. However, controversies exist about the definition of anatomical resection and the requisite width of negative margins. Consequently, a consensus on these issues is far from being achieved in the specialized surgical community. Review of the literature and author's discernment support AR for HCC larger than $2 \mathrm{~cm}$, and tumor exposure when the tumor is in contact with major vessels. Therefore, tumor exposure is not a contradiction to an AR properly carried out.
\end{abstract}




\section{Introduction}

Hepatocellular carcinoma (HCC) is one of the five most common malignancies worldwide, and its incidence is increasing in many countries [1]. Apart from liver transplantation, hepatic resection is the most potentially curative locoregional treatment for HCC. Improvements in perioperative care and refinements in surgical techniques have significantly increased the safety and survival rates of hepatic resection in the last few decades [2, 3]. However, the high rate of postoperative recurrence, $50-70 \%$ at 3 years, remains a major concern $[4,5]$. Such recurrences usually occur in the liver owing to the high propensity of HCC to invade portal vein branches [6], and to the underlying liver cirrhosis, which is the ideal background for the development of HCC. Two pivotal surgical techniques are usually recommended to reduce postoperative recurrence: anatomical resection (AR)[7], and achievement of negative margins $[8,9]$. However, in the surgical community, some confusion still exists about these issues, particularly, about their definitions and for the rules governing their practical application. Therefore, this study aimed to review these two techniques of hepatic resection for HCC.

\section{Anatomical Resection (AR)}

AR is usually recommended because the removal of the portal vascular bed containing a tumor is theoretically expected to be effective from an oncological perspective. The rationale for this is that removal of the vascular bed will ensure the removal of any potential satellite tumors in the liver, which may have risen because of the tumor's tendency to invade the portal veins [6]. To achieve an optimal compromise between the need for the complete removal of the area occupied by the tumor and the need to spare the liver parenchyma, Makuuchi et al. proposed systematic subsegmentectomy in 1985 [7], and successfully applied it, mainly in Japan, with excellent results [10]. Given the technical skill demanded by this procedure, which involves free-hand ultrasound-guided puncture of thin portal branches, an alternative method of segmental or subsegmental AR has recently been introduced: the ultrasoundguided finger compression technique [11]. Any other technique that does not aim to precisely identify the afferent portal pedicle and the segmental or subsegmental territory it supplies should be considered a non-anatomical resection (NAR), regardless of whether it is associated with minor or major parenchymal removal. This is a crucial point that should be considered when comparing different studies on segmental or subsegmental AR and NAR for HCC. When the surgical technique is not detailed, the results may be biased by significant conceptual and technical issues, which make the conclusions invalid.

As a partial consequence of this inadequate comparison, it remains unclear whether hepatectomy performed in clinical practice should involve AR or NAR. No prospective randomized trials have been available to date, and two meta-analyses on the topic have reported conflicting findings $[12,13]$. Moreover, a recent meta-regression analysis showed that even after adjusting for some important covariates, the available studies on AR and NAR could not be easily compared [14]. A review of the most relevant studies published on this topic in the last decade is presented in table 1 [10,15-20]. Most of the studies report a trend of better 5 -year overall survival in the AR group than in the NAR group; in particular, it seems that the anatomical approach is advantageous mainly for lesions measuring $>2 \mathrm{~cm}$ and $<5 \mathrm{~cm}$ [17, $18,20]$. However, because comparisons of AR and NAR remain biased owing to technical issues and differences in cirrhosis, etiology, and tumor presentation, the superiority of AR over NAR could not be definitively determined. 
Table 1. Review of the literature on AR vs. NAR for HCC in the last decade

\begin{tabular}{cccccc}
\hline \multicolumn{1}{c}{ Author, year } & Groups & Patients & Mortality (\%) & 5-year overall survival & Conclusions \\
\hline Regimbeau, 2002 & AR & 30 & 7 & 54 & AR \\
& NAR & 34 & 6 & 35 & \\
Hasegawa, 2005 & AR & 156 & 0 & 66 & AR \\
& NAR & 54 & 0 & 35 & NAR \\
Kaibori, 2006 & AR & 34 & 2.9 & 52.7 & \\
& NAR & 213 & 1.9 & 46.2 & AR if $>2$ cm \\
Wakai, 2007 & AR & 95 & 2 & 67 & \\
& NAR & 63 & 6 & 59 & AR if $2-5 \mathrm{~cm}$ \\
Eguchi, 2008 & AR & 2267 & 0.7 & 65.5 & \\
& NAR & 3514 & 0.8 & 62.4 & NAR \\
Tanaka, 2008 & AR & 83 & 0 & 54 & AR if $>2$ cm \\
& NAR & 42 & 0 & 61 & \\
Yamazaki, 2010 & AR & 111 & 1.8 & 71 & \\
& NAR & 98 & 0 & & \\
\hline
\end{tabular}

Table 2. Review of the literature on surgical margins for HCC

\begin{tabular}{lcclc}
\hline \multicolumn{1}{c}{ Author, year } & $\begin{array}{c}\text { Number of } \\
\text { patients }\end{array}$ & Margin (mm) & Study Design & Findings \\
\hline Chau, 1997 & 165 & 10 & Retrospective & Better DFS ( $>10 \mathrm{~mm})$ \\
Ochiai, 1999 & 68 & 0 & Retrospective & No differences \\
Poon, 2000 & 288 & 10 & Retrospective & No differences \\
Matsui, 2007 & 465 & 0 & Retrospective & No differences \\
Shi, 2007 & 84 vs. 85 & 20 & Randomized & Better OS and DFS ( $>20 \mathrm{~mm})$ \\
\hline \multicolumn{2}{c}{ OS = Overall survival; DFS = Disease free survival. } & & \\
\hline
\end{tabular}

\section{Surgical Margins}

The effect of surgical margin status on the survival of patients with HCC has been studied, but controversies persist among surgeons. Some authors have reported that margins smaller than $1 \mathrm{~cm}$ or even $2 \mathrm{~cm}$ negatively affect long-term survival $[8,9]$, while others have found opposite results, stating that even 0 -mm margins are acceptable [21-23].

A review of the literature on this topic has been presented in table 2: only one randomized controlled study, published by Shi et al. [9] in 2007, is available on this issue. The authors compared HCC patients with 1-cm margins versus those with 2-cm margins and observed a lower recurrence rate in the latter group. The high rate of local recurrence (29\%), which is inconsistent with other larger series, and the unclear description of AR and NAR remain major drawbacks of that study. Regarding the latter point, the authors considered certain cri- 
teria that should not have been included: tumor location in terms of depth into the liver and tumor location at the edge between two adjacent segments. These two conditions are not contraindications to AR. In these conditions, multiple punctures or compressions of subsegmental/segmental portal branches should be performed to anatomically demarcate the area to be removed.

A concept that should be stressed in discussions of surgical margin status is the relationship between the width of tumor-free margin and tumor size. The risk of satellites increases proportionally with tumor size; in HCCs larger than $2.5 \mathrm{~cm}$, the risk of microsatellites located more than $5 \mathrm{~mm}$ away from the tumor burden becomes significant [24]. Therefore, a clear margin should be achieved in the case of tumors larger than $2.5 \mathrm{~cm}$. These findings are consistent with the observation that in the case of HCCs smaller than $2 \mathrm{~cm}$, similar local control can be obtained using either the ablation technique or hepatic resection [25]. However, this should not act as a confounding finding when attention is focused on 0 -mm margins at the site of contact between the tumor and a major vessel, whether a glissonian pedicle or a hepatic vein. Under these circumstances, tumor exposure on the cut surface, even when the HCC is larger than $2.5 \mathrm{~cm}$, is acceptable; the possibility of microsatellites is obviously nil at this site and with appropriate surgery under intraoperative ultrasound guidance, the risk of local recurrence is negligible [26, 27]. Conversely, sacrificing the vessels could result in major parenchymal removal and increased surgical risk [28, 29].

\section{AR and Tumor Exposure}

From the aforementioned text, it can be considered that the performance of AR does not depend on the achievement of negative margins. Complete microsatellite removal depends on the complete removal of the tumor-containing part, i.e., the entire vascular bed supplying the lesion. An indirect proof is the finding that AR impacts prognosis in patients with tumors larger than $2 \mathrm{~cm}[17,18,20]$, or in other words, when the HCC has a higher risk of being associated with microsatellites [24] and when ablation is less efficient in providing local control [25]. However, the removal of an entire hepatic segment does not ensure the prevention of tumor exposure. For instance, in the case of an HCC that is located in segment 8 and is in contact with the right and middle hepatic veins at the caval confluence, a full AR of segment 8 will expose on the cut surface the right and middle hepatic veins; the specimen at the level of the detached contact between the HCC and the hepatic veins should have exposed the tumoral surface. As mentioned earlier, the possibility of microsatellites at this site is nil, and the risk of local recurrence becomes negligible if an adequate technique is meticulously applied under intraoperative ultrasound guidance [26, 27]. However, sparing of the vessel by means of tumor-vein detachment minimizes the excision of the liver parenchyma, and it is well established that the prognosis of HCC patients depends much more on the residual liver volume than on the width of the surgical margin [29].

Thus, conceptually, any new lesion occurring in the adjacent segments during the postoperative follow-up period should not be considered as an undetected satellite not removed during surgery, but rather as a distant metastasis (fig. 1). Given the intrahepatic diffusion of HCC through the portal vein system, any metastatic lesion growing in a segment other than the one in which the primary tumor originated should be considered a distant metastatic tumor, regardless of its physical distance from the segment containing the primary HCC. 


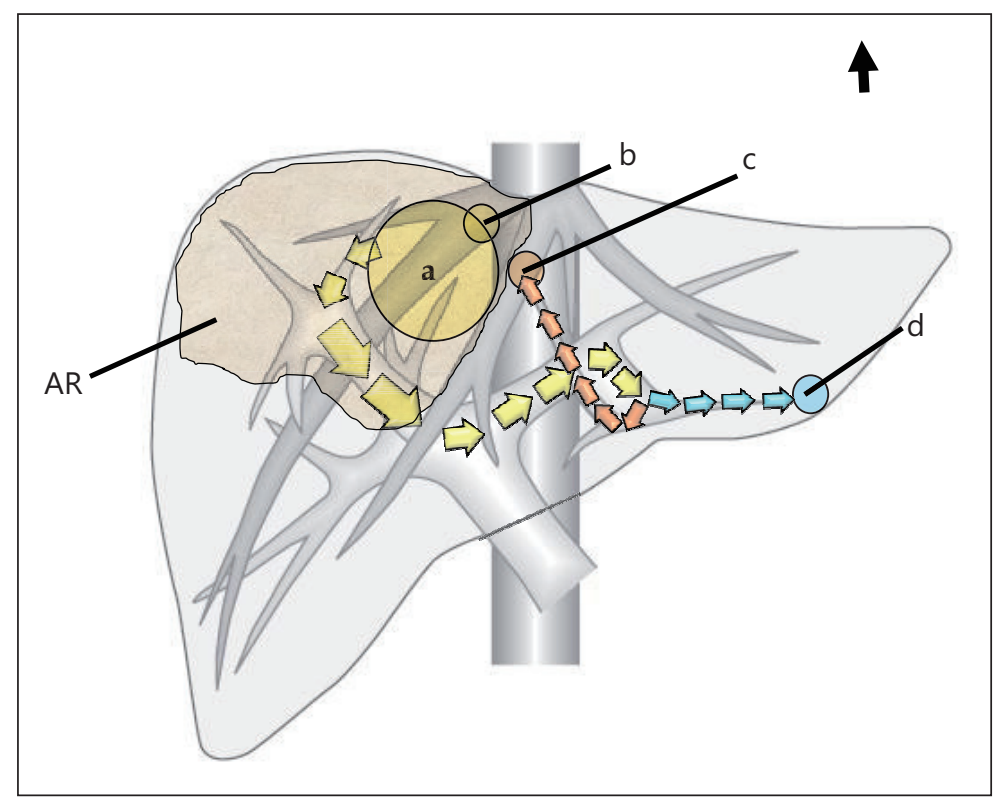

Fig. 1. Given the propensity of HCC to undergo intrahepatic dissemination through the portal branches (arrows), we recommend that any lesion not supplied by the same segmental portal branch as the main tumor (a) should be considered a distant metastasis rather than a satellite tumor (b), regardless of its location, whether adjacent to (c) or distant from (d) the main tumor and the resection area.

\section{Conclusions}

The success of hepatic resection for HCC relies on the accurate balance between the functional reserve of the residual liver and the best local control of the tumor. The review presented herein of the literature, together with the authors' discernment, does not support either AR or large surgical margins a priori. The better results obtained with AR than with NAR cannot be definitively attributed to the superior oncological control of AR, although this is theoretically reasonable. The role of AR is probably not very important in the case of HCCs smaller than $2 \mathrm{~cm}$, and moreover, the surgical approach in general is increasingly not being used to treat in such cases. For lesions larger than $2 \mathrm{~cm}$, it seems reasonable that a tumor-free margin of at least $0.5 \mathrm{~cm}$ be obtained, unless the tumor is not in contact with a major vessel. However, in this last circumstance, the risk of local recurrence is low, and the outcomes of different technical solutions to spare the vessel should be compared with the worse short- and longterm outcomes of vessel resection before a major hepatectomy is carried out. We believe that tumor exposure is not a contradiction to an AR that is properly carried out with the complete removal of the tumor-containing segment or subsegment. To ensure surgical safety, radical oncological resection with narrow margins, and anatomical, but limited, liver resection, a surgeon's skill in intraoperative ultrasonography is mandatory.

\section{References}

1 El-Serag HB, Davila JA, Petersen NJ, McGlynn KA: The continuing increase in the incidence of hepatocellular carcinoma in the United States: an update. Ann Intern Med 2003;139:817-823.

2 Torzilli G, Makuuchi M, Inoue K, et al: No-mortality liver resection for hepatocellular carcinoma in cirrhotic and noncirrhotic patients: is there a way? A prospective analysis of our approach. Arch Surg 1999;134:984992. 
3 Cucchetti A, Zanello M, Cescon M, et al: Improved diagnostic imaging and interventional therapies prolong survival after resection for hepatocellular carcinoma in cirrhosis: the University of Bologna experience over 10 years. Ann Surg Oncol 2011;18:1630-1637.

4 Fan ST, Ng I, Poon R, et al: Hepatectomy for hepatocellular carcinoma: the surgeon's role in long-term survival. Arch Surg 1999;134:1124-1130.

5 Imamura H, Matsuyama Y, Miyagawa Y, et al: Prognostic significance of anatomical resection and des- $\gamma-$ carboxy prothrombin in patients with hepatocellular carcinoma. Br J Surg 1999;86:1032-1038.

6 Yuki K, Hirohashi S, Sakamoto M, et al: Growth and spread of hepatocellular carcinoma: a review of 240 consecutive autopsy cases. Cancer 1990;66:2174-2179.

7 Makuuchi M, Hasegawa H, Yamazaki S: Ultrasonically guided subsegmentectomy. Surg Gynecol Obstet 1985;161:346-350.

8 Chau GY, Lui WY, Tsay SH, et al: Prognostic significance of surgical margin in hepatocellular carcinoma resection: an analysis of 165 Childs' A patients. J Surg Oncol 1997;66:122-126.

9 Shi M, Guo RP, Lin XJ, et al: Partial hepatectomy with wide versus narrow resection margin for solitary hepatocellular carcinoma: a prospective randomized trial. Ann Surg 2007;245:36-43.

10 Hasegawa K, Kokudo N, Imamura H, et al: Prognostic impact of anatomic resection for hepatocellular carcinoma. Ann Surg 2005;242:252-259.

11 Torzilli G, Procopio F, Cimino M, et al: Anatomical segmental and subsegmental resection of the liver for hepatocellular carcinoma: a new approach by means of ultrasound-guided vessel compression. Ann Surg 2010;251:229-235.

12 Chen J, Huang $\mathrm{K}, \mathrm{Wu}$ J, et al: Survival after anatomic resection versus nonanatomic resection for hepatocellular carcinoma: a meta-analysis. Dig Dis Sci 2011;56:1626-1633.

13 Zhou Y, Xu D, Wu L, Li B: Meta-analysis of anatomic resection versus nonanatomic resection for hepatocellular carcinoma. Langenbecks Arch Surg 2011;396:1109-1117.

14 Cucchetti A, Cescon M, Ercolani G, et al.: A Comprehensive Meta-regression Analysis on Outcome of Anatomic Resection Versus Nonanatomic Resection for Hepatocellular Carcinoma. Ann Surg Oncol 2012 ;19:3697-3705.

15 Regimbeau JM, Kianmanesh R, Farges 0, et al: Extent of liver resection influences the outcome in patients with cirrhosis and small hepatocellular carcinoma. Surgery 2002;131:311-317.

16 Kaibori M, Matsui Y, Hijikawa T, et al: Comparison of limited and anatomic hepatic resection for hepatocellular carcinoma with hepatitis C. Surgery 2006;139:385-394.

17 Wakai T, Shirai Y, Sakata J, et al: Anatomic resection independently improves long-term survival in patients with T1-T2 hepatocellular carcinoma. Ann Surg Oncol 2007;14:1356-1365.

18 Eguchi S, Kanematsu T, Arii S, et al: Liver Cancer Study Group of Japan. Comparison of the outcomes between an anatomical subsegmentectomy and a non-anatomical minor hepatectomy for single hepatocellular carcinomas based on a Japanese nationwide survey. Surgery 2008;143:469-475.

19 Tanaka K, Shimada H, Matsumoto C, et al: Anatomic versus limited nonanatomic resection for solitary hepatocellular carcinoma. Surgery 2008;143:607-615.

20 Yamazaki 0, Matsuyama M, Horii K, et al: Comparison of the outcomes between anatomical resection and limited resection for single hepatocellular carcinomas no larger than $5 \mathrm{~cm}$ in diameter: a single-center study. J Hepatobiliary Pancreat Sci 2010;17:349-358.

21 Ochiai T, Takayama T, Inoue K, et al: Hepatic resection with and without surgical margins for hepatocellular carcinoma in patients with impaired liver function. Hepatogastroenterology 1999;46:1885-1889.

22 Poon RT, Fan ST, Ng IO, et al: Significance of resection margin in hepatectomy for hepatocellular carcinoma: A critical reappraisal. Ann Surg 2000;231:544-551.

23 Matsui Y, Terakawa N, Satoi S, et al: Postoperative outcomes in patients with hepatocellular carcinomas resected with exposure of the tumor surface: clinical role of the no-margin resection. Arch Surg 2007;142:596-602.

24 Sasaki A, Kai S, Iwashita Y, et al: Microsatellite distribution and indication for locoregional therapy in small hepatocellular carcinoma. Cancer 2005;103:299-306.

25 Livraghi T, Meloni F, Di Stasi M, et al: Sustained complete response and complications rates after radiofrequency ablation of very early hepatocellular carcinoma in cirrhosis: Is resection still the treatment of choice? Hepatology 2008;47:82-89.

26 Torzilli G, Montorsi M, Donadon M, et al: "Radical but conservative" is the main goal for ultrasonographyguided liver resection: prospective validation of this approach. J Am Coll Surg 2005;201:517-528.

27 Torzilli G, Montorsi M, Del Fabbro D, et al: Ultrasonographically guided surgical approach to liver tumours involving the hepatic veins close to the caval confluence. Br J Surg 2006;93:1238-1246.

28 Poon RT, Fan ST, Wong J: Risk factors, prevention, and management of postoperative recurrence after resection of hepatocellular carcinoma. Ann Surg 2000;232:10-24.

29 Bilimoria MM, Lauwers GY, Doherty DA, et al: International Cooperative Study Group on Hepatocellular Carcinoma. Underlying liver disease, not tumor factors, predicts long-term survival after resection of hepatocellular carcinoma. Arch Surg 2001;136:528-535. 